\title{
Incorporating cultural values and use of technology platforms may help outreach Asian Americans in cancer clinical trial participation
}

Angela Sun*1, Joyce Cheng ${ }^{1}$, Julie H.T. Dang ${ }^{2}$, Charlene Cuaresma ${ }^{3}$, Annalyn Valdez-Dadia ${ }^{4}$, Rachel J. Mesia ${ }^{5}$, Penny Lo $^{6}$, Edward A. Chow ${ }^{7}$, Vanessa Ho ${ }^{1}$, Tung T. Nguyen ${ }^{8}$, Moon S. Chen, Jr. ${ }^{2}$

${ }^{1}$ Chinese Community Health Resource Center (CCHRC), San Francisco, USA

${ }^{2}$ UC Davis Comprehensive Cancer Center, University of California, Davis, Sacramento, USA

${ }^{3}$ University of Hawai'l at Mānoa, Honolulu, USA

${ }^{4}$ Department of Community Health Sciences, UCLA Fielding School of Public Health, Los Angeles, USA

${ }^{5}$ Stanford Cancer Institute, Palo Alto, USA

${ }^{6} \mathrm{Hmong}$ Women's Heritage Association (Out of Business), USA

${ }^{7}$ Jade Health Care Medical Group, San Francisco, USA

${ }^{8}$ Division of General Internal Medicine, University of California, San Francisco, USA

Received: April 11, 2018

DOI: $10.5430 /$ ijh.v4n2p34
Accepted: June 19, 2018

Online Published: June 26, 2018

URL: https://doi.org/10.5430/ijh.v4n2p34

\begin{abstract}
Asian Americans are the fastest growing ethnic group in U.S. However, they represent only $1.7 \%$ of U.S. cancer clinical trial participants. This pilot study describes findings on barriers, promoters and recommendations related to cancer clinical trial participation from Asian Americans. The research team conducted 3 focus groups comprised of 21 community members and 4 key informant interviews with healthcare providers. Qualitative methodology was used to identify themes about cancer clinical trial participation. Barriers and promoters were categorized based on themes identified and previous study findings. Eight major themes and 5 recommendations were identified from the focus group data. Five major themes and 7 recommendations were identified from the key informant data. Asian Americans' decision to participate in cancer clinical trials is largely influenced by their cultural values and practices such as altruism and family-based decision making process. Technology platforms provide promising venues to reach Asian Americans. Family-based decision making process, altruism, and the use of technology platforms may need to be considered when outreaching to Asian Americans on cancer clinical trial participation.
\end{abstract}

Key Words: Cancer clinical trials, Asian American, Outreach, Barriers and promoters, Cultural practice, Technology

\section{INTRODUCTION}

Participation of racial/ethnic minorities in cancer clinical trials is a glaring health disparity affecting all U.S. populations of color. ${ }^{[1,2]}$

Asian Americans are the fastest growing racial group in the
U.S. and account for $6.3 \%$ of the population. ${ }^{[3]}$ Chinese $(22.5 \%)$ and Filipino (18.5\%) are among the largest Asian American groups. ${ }^{[4]}$ Hmong Americans comprise about 1.5\% of Asian Americans. ${ }^{[5]}$ Fifty nine percent of Asian Americans are foreign born and 36\% speak English less than "very well". ${ }^{[6]}$

*Correspondence: Angela Sun, PhD, MPH; Email: angelas@ chasf.org; Address: 728 Pacific Avenue Suite 115, San Francisco, CA 94133, USA. 
Cancer is the leading cause of death among Asian Americans. ${ }^{[7]}$ However, only $1.7 \%$ of cancer clinical trial participants are Asian Americans and Pacific Islanders (AAPI). ${ }^{[8]}$ Addressing these disparities in cancer clinical trials is a pressing national priority. ${ }^{[1,7]}$ Studies on Asian Americans have shown evidence of cultural differences in belief and attitude, ${ }^{[9,10]}$ healthcare decision-making ${ }^{[11,12]}$ and perception regarding clinical trial participation. ${ }^{[1,13-19]}$ Despite growing body of findings related to clinical trial participation, enrollment into clinical trials from Asian Americans remains low. ${ }^{[8]}$ This paper aims to describe findings from a pilot study on barriers, promoters of and recommendations for cancer clinical trial participation among Chinese, Filipino and Hmong Americans.

\section{MethodS}

This pilot study was conducted by Asian community based organizations and academia located in Hawaii, San Francisco and Sacramento. The research team consisted of representatives from each organization. The team participated in monthly teleconferences to develop the interview guide, create a recruitment plan and identify themes from the interviews. This study utilized qualitative methodology through focus groups with participants from Chinese, Filipino, and Hmong Asian American descent and interviews of key informants who cared for those subgroups. The study was approved by the Institutional Review Board of the Ethical and Independent Review Services of Corte Madera, California.

\subsection{Qualitative interviews: Community member focus groups}

Each organization was instructed to conduct a focus group among 6 to 8 targeted community members in the respective native language of the community, except for the Filipino community since the majority of Filipinos were proficient in English.

The inclusion criteria for focus group participants included self-reporting as a foreign born Asian American, being at least 18 years old, and speaking Chinese, Filipino (Tagalog, Ilocano and or English) or Hmong as a primary language. Cancer status and years of residence in U.S. were not considered. Individuals who participated in the interview guide testing were not eligible to participate in the focus group.

The participants for the focus group were recruited through a convenience sampling method by the community organization at each location. Using flyers and one-on-one contact, participants were recruited from their local faith-based communities, hospitals and clinics, healthcare offices, community centers, cancer support groups, and ethnic media readership.
The research team developed the interview guide to cover domains related to cancer clinical trial participation including perception, awareness, barriers, promoters and outreach approaches. The community organization at each location translated the interview guide into the respective language of its target population and tested the guide among the intended communities. Each of the focus groups was conducted at a local community center, which provided an environment that was culturally familiar to and comfortable for the participants. A trained bilingual facilitator, who was fluent in English and the targeted native languages or dialects, used the interview guide to conduct the focus group in the native language of the community except for the Filipino community, which was conducted in English. Prior to the beginning of each focus group, participants provided verbal consent to participate. All of the focus group interviews were audio recorded. A standardized demographic survey was administered to all participants. The survey items included age, gender, descent, annual household income, education level, stage of cancer, and if any family member was living with cancer. A monetary incentive of $\$ 15$ was provided to each participant upon completion of the focus group interview. Each focus group was 90 minutes in duration.

\subsection{Qualitative interview: Health care providers as key informants}

The key informant interview targeted 4 to 6 oncologists who cared for the target subgroups of Asian American patients. Other specialists acted as the key informant if an oncologist was not available at the respective location. The key informants were recruited from the healthcare systems of each targeted community. The research team developed the key informant interview guide to cover domains such as their willingness to participate in cancer clinical trials, perceived barriers and outreach approaches. Using the interview guide, the trained facilitator conducted the interview in English. The length of the interview was approximately one hour and incentives were not provided to the key informants. Key informants provided verbal consent to participate prior to the interview. Qualitative data from key informant interviews were captured through interview notes.

\subsection{Qualitative data analysis}

All interview transcripts were analyzed by utilizing the Qualitative Content Analysis Method. ${ }^{[20]}$ All transcripts of the focus group interviews were transcribed into English from the native languages of each group, with the exception of the Filipino focus group. All interview transcripts and notes were reviewed independently by each organization's representative who then identified and labeled themes. The research team members subsequently communicated over several confer- 
ence calls to discuss and compare one another's independent findings as well as detect new themes not previously noted. Any inconsistencies or disagreements in interpretation of certain themes were clarified through further discussions in order to validate the findings. Themes were categorized into barriers, promoters and recommendations for cancer clinical trial participation.

\section{RESUlts}

\subsection{Focus groups}

A total of 3 focus groups consisting of 21 community members with ages ranging from 50-88 years participated in the study. Of the total participants: 6 were Chinese, 6 were Filipino, and 9 were Hmong. Sixty percent of participants were female, $65 \%$ had less than $\$ 25,000$ annual household income, about one-third have had high school education level or lower, $20 \%$ were cancer survivors, $20 \%$ had family members who were diagnosed with cancer; and $10 \%$ were undergoing cancer treatment. The remaining $50 \%$ of the focus group participants were Asian Americans with neither a history of cancer nor family members with cancer.

\subsubsection{Themes identified by focus group participants}

Many factors can serve as barriers and/or promoters for the participation in clinical trials.

Tables 1 and 2 summarized the themes that serve as barriers and promoters identified by focus group participants. We found the following to be barriers to clinical trial participation from the participants' focus groups. These barriers were also aligned with previous studies: lack of knowledge and understanding of the meaning and purpose of clinical trials, ${ }^{[14,15,17,19]}$ fear associated with clinical trial, ${ }^{[17,19]}$ lack of culturally and linguistically appropriate materials and education about clinical trials. ${ }^{[14,15,17,19]}$ Asian culture defers decision making to family members. ${ }^{[1,12,15,17,19]}$ Positive or supportive family-based health decision making dynamics was found to be a promoter for clinical trial participation, [17] while negative family dynamic or opposition in clinical trial participation from a family member was found to be a barrier to participation.

Table 1. Themes that serve as barriers identified by focus group participants

\begin{tabular}{|c|c|c|}
\hline Theme & Description & Responses \\
\hline $\begin{array}{l}\text { Lack of } \\
\text { knowledge } \\
\text { and } \\
\text { understanding } \\
\text { of the } \\
\text { meaning of } \\
\text { clinical trials }\end{array}$ & $\begin{array}{l}\text { When asked "what do you think of when you } \\
\text { hear the term clinical trial", some participants } \\
\text { have expressed that this is the first they heard } \\
\text { of the term "clinical trials" and a few other } \\
\text { participants seemed to have difficulties } \\
\text { distinguishing between treatments received at } \\
\text { clinical trials and treatments received at } \\
\text { doctor's office or hospital. }\end{array}$ & $\begin{array}{l}\text { I don't know what is clinical trial. - Hmong } \\
\text { This is the first time I heard about the term “clinical trials” and I don't } \\
\text { know anything about it. - Filipino } \\
\text { It's about seeing doctor and getting treatment. - Chinese } \\
\text { I think of going to hospital for treatment. - Chinese }\end{array}$ \\
\hline $\begin{array}{l}\text { Fear } \\
\text { associated } \\
\text { with clinical } \\
\text { trial } \\
\text { participation }\end{array}$ & $\begin{array}{l}\text { Participants expressed that "fear" was a barrier } \\
\text { to clinical trial participation. For example- fear } \\
\text { of the unknown, being assigned to the placebo } \\
\text { group, treatment side effects, possibility that } \\
\text { new medicine will not improve their current } \\
\text { condition and being a guinea pig. }\end{array}$ & $\begin{array}{l}\text { People would think twice about participating in clinical trials because of } \\
\text { potential side effects. - Chinese } \\
\text { First come to mind, to me, would be me being the guinea pig. You } \\
\text { know, trying out new things on me as an individual. - Filipino }\end{array}$ \\
\hline $\begin{array}{l}\text { Lack of } \\
\text { understanding } \\
\text { on the } \\
\text { purpose, } \\
\text { benefits and } \\
\text { protocol of } \\
\text { clinical trial } \\
\text { participation }\end{array}$ & $\begin{array}{l}\text { The majority of the participants said that } \\
\text { information on clinical trials were not available } \\
\text { and/or not easy for laymen to comprehend such } \\
\text { as its purpose, benefits and protocol. The } \\
\text { majority of Chinese participants thought that if } \\
\text { participants are placed in the placebo arm of } \\
\text { the clinical trial, they would not receive any } \\
\text { treatment. }\end{array}$ & $\begin{array}{l}\text { My doctor asked me to sign the consent form, but didn't quite explain } \\
\text { about the protocol of the trial. At that moment, my wife was strongly } \\
\text { against participation in a clinical trial, leading to disputes within our } \\
\text { family. - Chinese } \\
\text { Hmong people are fearful of clinical trials because they don't } \\
\text { understand the purpose, options and the entire process and the need of } \\
\text { participating in a study. - Hmong } \\
\text { It doesn't matter which group I am assigned to, as long as I receive } \\
\text { treatment. When you have cancer, it makes no sense if you're just under } \\
\text { observation and receive no treatment ( } 5 \text { participants agreed). - Chinese }\end{array}$ \\
\hline $\begin{array}{l}\text { Lack of } \\
\text { culturally and } \\
\text { linguistically } \\
\text { appropriate } \\
\text { materials and } \\
\text { education } \\
\text { about clinical } \\
\text { trials }\end{array}$ & $\begin{array}{l}\text { All groups voiced the need for culturally and } \\
\text { linguistically appropriate materials on clinical } \\
\text { trials. }\end{array}$ & $\begin{array}{l}\text { Lack of available information about cancer clinical trials that's why I } \\
\text { don't understand what it really is. It would be better for health care } \\
\text { professional to give advice and answer questions in Chinese. - Chinese } \\
\text { Not understanding the definition of clinical trial and there isn't enough } \\
\text { vocabulary in Hmong to describe the clinical trial. - Hmong } \\
\text { For the majority of our Filipino community bilingual materials would be } \\
\text { helpful because especially the older generation, always want to learn in } \\
\text { their own language. - Filipino }\end{array}$ \\
\hline
\end{tabular}


Table 2. Themes that serve as promoters identified by focus group participants

\begin{tabular}{ll}
\hline Theme & Description \\
\hline $\begin{array}{l}\text { Outcome of trials } \\
\text { would potentially } \\
\text { benefit me, } \\
\text { family or others }\end{array}$ & $\begin{array}{l}\text { When participants perceive that the outcomes of cancer } \\
\text { clinical trials can potentially benefit themselves, their } \\
\text { family or their communities, they would more likely to } \\
\text { participate in available cancer clinical trials. }\end{array}$ \\
& $\begin{array}{l}\text { Positive changes in attitude towards clinical trials were } \\
\text { observed among Chinese and Filipino focus group } \\
\text { participants after the definition of cancer clinical trials }\end{array}$ \\
$\begin{array}{l}\text { Clear definition } \\
\text { of cancer clinical } \\
\text { trials }\end{array}$ & $\begin{array}{l}\text { was introduced. One of the participants indicated that } \\
\text { s/he previously refused to participate in clinical trials } \\
\text { when asked, but after learning about the definition of } \\
\text { clinical trials, the participant was open to participate. }\end{array}$
\end{tabular}

Responses

If the clinical trial medication improves my condition, it would help other people. - Hmong

Clinical trial participation is important because I can make a contribution to the society. - Chinese

I just heard about the definition of clinical trials. It is a good one, now I'm willing to participate so that more lives will be spared. - Filipino

Before hearing the definition, I didn't really trust medicines because I thought they are poisonous. I always associate chronic illness with incurable cancer. But now I come across this definition, I start to realize the importance of treatment and prevention. - Chinese

Family opinions, personal belief and values of life are also

Asian Americans consider their family members' point

Family-based health decision making dynamics of views when making decision about clinical trial participation. All participants conveyed that participation in clinical trials were influenced by their family's opinion and attitude towards clinical trial participation.

Advanced stage cancer diagnosis may promote participation in cancer clinical trials

The targeted Asian participants of Chinese and Hmong descent were more receptive to participating in clinical trials if they are in poor health. They would be open to participate in clinical trials if they were diagnosed with advanced stage of cancer and all available conventional treatment options were exhausted.

Health care professionals such as doctors, nurses, community health educator, researchers, social workers, psychologist, including physicians and nurses, were identified as preferred health messengers to deliver information on clinical trials.

Preferred health messengers

Participants identified conventional and technology-related venues that they currently use to

Preferred outreach venues important. Children's and spouses' opinions can also affect my decisions. - Chinese

Educate the person who is sick and also the family so they understand the purpose of the study, options, and whole process. - Hmong

When I consider to participate in cancer clinical trials, I care about my family member's support. - Filipino

If the person receives "death sentence", then they would try to participate. To be considered "death sentence", one must have 3 to 6 remaining months to live. - Chinese

People who are sick should participate in clinical trials because there is nothing to lose. - Hmong

I would trust my doctor to know what is good for me. And so, if he assigns me to placebo group, I don't think he will do it to harm me. But if I have a choice, I would like to be in the treatment group. My doctor knows what's best for me; I would ask him what he thinks. - Filipino

If the doctors, nurses, or medical personnel, community health educator ask me to participate in clinical trials, I will consider it. What doctors say will give me hope and make me think twice about my conditions. - Chinese

I believe bilingual physicians who speak Ilokano or Tagalog would be excellent messengers for Filipinos in their community. - Filipino

Provide more education through video such as via YouTube. Teach us so we can help teach others to have more trust and be more open. - Hmong

Many Chinese are using the credible websites for health information. - Chinese

Chinese radio is effective because people can listen to it while driving. - Chinese

I learn about news related to health through television, as well as internet. - Filipino

A clear definition of cancer clinical trials was found to facilitate understanding and acceptance of clinical trials participation. Understanding the benefits and protocol of clinical trial and having an advanced stage cancer diagnosis were also found to promote participation. As suggested by focus group participants, when individuals see positive outcome of trials that could potentially benefit themselves, their family or others, they would be more likely to participate. Additionally, focus group participants identified healthcare professionals as the preferred health messengers and technology platforms such as credible websites, YouTube, and ethnic media were preferred outreach venues. 


\subsubsection{Recommendations from focus group participants}

Messages on clinical trial participation outreach be focused on the benefits of outcomes to patient, family and /or their communities; education material be culturally and linguistically appropriate for the target patient population; healthcare providers should educate patient and their family members regarding clinical trials and involve patients' family members in healthcare decision making process; health messengers be healthcare professionals; technology platforms such as credible websites, YouTube, and ethnic media be used to promote messages on clinical trial participation.

\subsection{Key informant interviews}

A total of 4 healthcare providers of Asian descent participated in the key informant interviews, of which 3 were oncologists and 1 was a hepatologist. Ages ranged from 50 to 65 years. Two were males and 2 were females.

\subsubsection{Themes identified by key informants}

Lack of in-language consent forms and clinical trial information for patients; clinical trial enrollment is complex and time consuming; patients' fear of possibility of being enrolled into a placebo and/or double placebo group; lack of funding for internal system to support clinical trial participation; lack of training on clinical trial for physicians during their fellowship/residency and training on cultural barriers related to clinical trial participation when communicating with Asian American cancer patients.

\subsubsection{Recommendations from key informants}

Patient education information should be brief, in the patients' native language and non-pharmaceutical branded; healthcare providers to begin the in-person dialogue about clinical trial participation with patients; utilize ethnic media campaign to raise awareness on clinical trials among Asian Americans; embed clinical trial information within other patient education topics; healthcare providers should seek training/continuing medical education on clinical trials and be aware of resources available on current clinical trials; healthcare providers should stay connected to clinical trial providers' networks and adapt a relevant, feasible and practical work flow to allow time to have dialogues with their patients about clinical trial participation enrollment.

\section{Discussion}

The majority of the themes and recommendations identified from this qualitative interviews are similar to previous studies. ${ }^{[1,12,14,15,17-19]}$ However, the incorporation of the themes on family decision-making process, cultural altruism and use of technology platforms to promote participation in clinical trials among Asian Americans may warrant further exploration.

\subsection{Family-centered healthcare decision-making pro-} cess

In part, low participation in clinical trials from Asian American Americans may reflect individuals' perceived conflicts between asserting self-autonomy and promoting family concordance in medical decision-making. The perception of family support in the participation of clinical trials would ameliorate this potential deterrent. Asian Americans would be more likely to participate in clinical trials if their assumed healthcare decision maker, such as the oldest son or a family member who is a healthcare professional, would encourage their participation in clinical trials. Therefore, it is essential for healthcare providers to identify and involve the family health decision maker(s) in the conversation about the possibility of participating in clinical trials. Educating the younger generation on clinical trials may also help to facilitate and encourage family members to make an informed decision on trial participation. However, when the family-centered healthcare decision making dynamic is not realized or incorporated appropriately in the outreach strategies, the familycentered decision making process could become a barrier to clinical trial participation as well.

\subsection{Altruism in the Asian culture}

Similar to previous studies, ${ }^{[8,18,19]}$ our finding confirms that altruism may serve as a promoter for cancer clinical trial participation.

Altruism or selflessness is the principle or practice of concern for the welfare of others. It is a traditional virtue in many cultures and religions such as Buddhism, Taoism and Confucianism $^{[10]}$ and Christianity. Most Filipino Americans are influenced by the teachings of Catholicism or Christianity. Many Hmong Americans share the traditions of Buddhism, Taoism and Confucianism. ${ }^{[21]}$ Taoism and Confucianism are both ancient styles of living which influence attitudes and behavior of most individuals who share these traditions. ${ }^{[22]}$ Confucianism and Taoism share common beliefs that each individual should act with virtue in all social matters to ensure order, harmony and unity within the family, community, country and universe. Their philosophies stress that good conduct will be rewarded with good health and a long life. ${ }^{[23]}$ Although many Chinese and Hmong Americans do not identify with the Catholic and Christian religions, Asian culture values altruism and holds in high regard the welfare of individuals, families and their associated communities.

To promote participation in clinical trials among Asian Americans, outreach programs should emphasize how the outcome of clinical trials can benefit the individuals, their families and communities, since altruism is a highly valued virtue by this ethnic group. 
4.3 Utilizing technology platforms as an outreach venue Our focus group participants and key informants suggested using technology platforms such as credible websites, YouTube, ethnic media as venues for outreach. Asian Americans have approximately $20 \%$ more broadband at home, laptop use, and wireless connectivity compared with the national averages, and utilization of social media is gaining popularity among Asian Americans. ${ }^{[24]}$ In a convenience sampling of 414 Chinese low income immigrants in San Francisco, we found that $75 \%$ had Internet access and $62 \%$ had a computer. ${ }^{[25]}$ In 2013, in a survey of 403 Chinese American immigrants ages 50 to 75 , we found that $52.1 \%$ had smartphones, $86 \%$ had Internet access at home, $72 \%$ used the Internet for health information, and $53 \%$ would like to learn how to use their smartphones to improve their health. ${ }^{[26]}$ These technology platforms could be effective venues to reach and educate communities about clinical trials. Future studies should explore the use of technology platforms as an outreach method for education on clinical trial participation.

\section{Conclusion}

This pilot study provided insights on barriers, promoters and recommendations related to cancer clinical trial participation from Asian Americans. Family-centered dynamics and cultural altruism should be recognized and incorporated into outreach strategies on cancer clinical trial participation to promote enrollment into trials from the Asian American communities. Use of technology platforms such as credible websites, YouTube, ethnic media may be an effective venue to perform outreach and deliver messages on cancer clinical trial participation, however, these platforms may warrant further exploration. The recommendations from community members and healthcare providers described in this study should be considered when designing outreach efforts to increase cancer clinical trial participation from Asian Americans. With the anticipated fast growing pace of the various Asian American racial groups in the U.S., the differences in cultural values and beliefs of each racial group should be considered when setting health policies and measures.

\section{Limitation}

The primary limitation of this pilot study is its small sample size. While this limits generalizability, the findings could provide insights on incorporating Asian cultural values and technology platform use in promoting cancer clinical trial participation among Asian Americans.

\section{ACKNOWLEDGEMENTS}

This paper was supported in part by a cooperative agreement from the National Cancer Institute to the National Center for Reducing Asian American Cancer Health Disparities, U54CA153499 and a grant from the National Institute on Minority Health and Health Disparities, 1U24MD006970. However, the content of this paper reflects those of the authors and their findings and do not necessarily represent the views of the National Institutes of Health.

\section{Conflicts of InTerest Disclosure}

The authors declare that they have no competing interests.

\section{REFERENCES}

[1] Ma GX, Seals B, Tan Y, et al. Increasing Asian American participation in clinical trials by addressing community concerns. Clin Trials. 2014; 11(3): 328-35. PMid:24603005. https ://doi .org/10.117 $7 / 1740774514522561$

[2] Chen MS, Jr., Lara PN, Dang JH, et al. Twenty years post-NIH Revitalization Act: enhancing minority participation in clinical trials (EMPaCT): laying the groundwork for improving minority clinical trial accrual: renewing the case for enhancing minority participation in cancer clinical trials. Cancer. 2014; 120 Suppl 7: 1091-6. PMid:24643646. https ://doi.org/10.1002/cncr. 28575

[3] Colby SL, Ortman JM. Projections of the Size and Composition of the U.S. Population: 2014 to 2060 [Internet]. 2015 Report No.: $\mathrm{P} 25-1143$.

[4] U.S. Census Bureau. American Community Survey 2015, 1-Year Estimates, Asian alone or in any combination [Internet]. 2015 Report No.: B02018.

[5] U.S. Census Bureau. The Asian popluation: 2010 census briefs [Internet]. 2012 Report No.: C2010BR-11.
[6] Pew Research Center. The Rise of Asian Americans [Internet]. 2012 [cited 2018 April 4]. 2013. Available from: http://www . pewsocialtrends . org/2012/06/19/the-ris e-of-asian-americans/

[7] American Cancer Society. Cancer Facts and Figures 2016. Atlanta, GA: American Cancer Society, 2016.

[8] Chen Jr. MS, Lara PN, Dang JHT, et al. Twenty years post-NIH revitalization act: renewing the case for enhancing minority participation in cancer clinical trials. Cancer. 2014; 120(7 Suppl 7): 1091-6. PMid:24643646. https ://doi.org/10.1002/cncr. 28575

[9] Coburn CL, Weismuller PC. Asian motivators for health promotion. J Transcult Nurs. 2012; 23(2): 205-14. PMid:22294332. https : //doi.org/10.1177/1043659611433869

[10] Johnson RC, Danko GP, Darvill TJ, et al. Cross-cultural assessment of altruism and its correlates. Personality and Individual Differences. 1989; 10(8): 855-68. https://doi.org/10.1016/0191 -8869 (89) $90021-4$

[11] McLaughlin LA, Braun KL. Asian and Pacific Islander cultural values: considerations for health care decision making. Health Soc 
Work. 1998; 23(2): 116-26. https://doi.org/10.1093/hsw/23 .2 .116

[12] Tung WC. Asian American's Confucianism-Based Health-Seeking Behavior and Decision-Making Process. Home Health Care Management and Practice. 2010; 22(7): 536-8. https ://doi .org/10.117 $7 / 1084822310376610$

[13] Alexander GA, Chu KC, Ho RC. Representation of Asian Americans in clinical cancer trials. Ann Epidemiol. 2000; 10(8 Suppl): S61-7. https ://doi.org/10.1016/S1047-2797(00)00198-8

[14] Lin JS, Finlay A, Tu A, et al. Understanding immigrant Chinese Americans' participation in cancer screening and clinical trials. J Community Health. 2005; 30(6): 451-66. PMid:16370055. https : //doi.org/10.1007/s10900-005-7280-5

[15] Tu SP, Chen H, Chen A, et al. Clinical trials: understanding and perceptions of female Chinese-American cancer patients. Cancer. 2005; 104(12 Suppl): 2999-3005. PMid:16247796. https://doi. org/10.1002/cncr. 21524

[16] Paterniti DA, Chen MSJ, Chiechi C, et al. Asian Americans and cancer clinical trials: a mixed-methods approach to understanding awareness and experience. Cancer. 2005; 104(12 Suppl): 3015-24. PMid:16247795. https://doi.org/10.1002/cncr. 21522

[17] Ma GX, Tan Y, Blakeney NC, et al. The impact of a communitybased clinical trial educational intervention among underrepresented Chinese Americans. Cancer Epidemiol Biomarkers Prev. 2014; 23(3): 424-32. PMid:24092627. https ://doi .org/10.1158/1055-996 5.EPI-13-0773

[18] George S, Duran N, Norris K. A systematic review of barriers and facilitators to minority research participation among African
Americans, Latinos, Asian Americans, and Pacific Islanders. Am J Public Health. 2014; 104(2): e16-31. PMid:24328648. https : //doi.org/10.2105/AJPH. 2013.301706

[19] Lee GE, Ow M, Lie D, et al. Barriers and facilitators for clinical trial participation among diverse Asian patients with breast cancer: a qualitative study. BMC Women's Health. 2016; 16: 43. PMid:27449505. https ://doi.org/10.1186/s12905-016-0319-1

[20] Cavanagh S. Content analysis: concepts, methods and applications. Nurse Res. 1997; 4(3): 5-16. https://doi.org/10.7748/nr19 $97.04 .4 .3 .5 . c 5869$

[21] Lee JHX, Matsuoka F, Yee E, et al. Asian American Religious Cultures. Santa Barbara: ABC-CLIO; 2015. 448-52 p.

[22] Dubs HH. The Development of Altruism in Confucianism. Philosophy East and West. 1951; 1(1): 48-55. https://doi.org/10. 230 $7 / 1396935$

[23] Compton's Learning Company. Chinese cultural studies: Philosophy and religion in China. Compton's Encyclopedia \& Fact-index1995.

[24] The Nielsen Company. State of the Asian American Consumer: Growing market, growing impact [Internet]. 2012 [cited 2018 April 4]. Available from: https ://www . aancinc.org/uploads/8/6/ 6/9/8669813/state_of_the_asian_american_consumer_r eport_from_the_nielsen_company_2012.pdf

[25] Sun A, Cheng J, Chan J, et al. Chinese Community eHealth Day as a venue to introduce inlanguage health information and technology trends among the older (50+) Chinese immigrant population. American Public Health Association 139th Annual Meeting and Exposition; Washington, DC.2011.

[26] Sun A. Chinese Community eHealth Day. 2013. 